\title{
Impact of Motor Speech Intervention on Neural Activity in Children with Speech Sound Disorders: Use of Magnetoencephalography
}

\author{
Vickie Y. Yu1 ${ }^{1}$, Darren S. Kadis ${ }^{2}$, Debra Goshulak ${ }^{3}$, Aravind K. Namasivayam ${ }^{4}$, \\ Margit Pukonen ${ }^{3}$, Robert M. Kroll ${ }^{3,4}$, Luc F. De Nil' ${ }^{4}$ Elizabeth W. Pang5,6* \\ ${ }^{1}$ Department of Communication Disorders and Sciences, California State University Northridge, California, USA \\ ${ }^{2}$ Division of Neurology and Pediatric Neuroimaging Research Consortium, Cincinnati Children's Hospital Medical Center, \\ Cincinnati, USA \\ ${ }^{3}$ The Speech \& Stuttering Institute, Toronto, Canada \\ ${ }^{4}$ Department of Speech-Language Pathology, University of Toronto, Toronto, Canada \\ ${ }^{5}$ Neurosciences and Mental Health, Sick Kids Research Institute, Toronto, Canada \\ ${ }^{6}$ Division of Neurology, Hospital for Sick Children, Toronto, Canada \\ Email: *elizabeth.pang@sickkids.ca
}

How to cite this paper: Yu, V.Y., Kadis, D.S., Goshulak, D., Namasivayam, A.K., Pukonen, M., Kroll, R.M., De Nil, L.F. and Pang, E.W. (2018) Paper Title. Journal of Behavioral and Brain Science, 8, 415-429. https://doi.org/10.4236/ibbs.2018.87026

Received: May 25, 2018

Accepted: July 2, 2018

Published: July 5, 2018

Copyright $(9) 2018$ by authors and Scientific Research Publishing Inc. This work is licensed under the Creative Commons Attribution-NonCommercial International License (CC BY-NC 4.0). http://creativecommons.org/licenses/by-nc/4.0/ (c) (i) (8) Open Access

\begin{abstract}
We present the novel use of a neuroimaging technique, magnetoencephalography (MEG), for examining therapy-related changes in neural activity during a speech and a non-speech motor task in children with speech sound disorders (SSD). Nine children (mean age $=4.2$ years) with SSD were scanned in the MEG before and after an eight-week course of intensive motor speech therapy. MEG tasks involved an oromotor and a syllable production task. MEG analyses identified significant post-therapy changes in brain regions related to oromotor control and speech production. Behavioral assessments showed significant improvements on measures of motor speech skills and articulation following intervention. This is the first demonstration of the ability of MEG to: 1) capture brain activations resulting from oromotor movements and simple syllable production in young children, and 2) capture brain changes related to speech therapy. As the findings from this study are promising, we discuss directions for the design of future studies to further examine specific neural dysfunctions in speech sound disorders.
\end{abstract}

\section{Keywords}

Speech Sound Disorders, Children, Intervention, Neuroimaging, Speech Production 


\section{Introduction}

The production of speech sounds requires the integration of several neural pathways and complex speech motor movements that require the control and coordination of multiple oral motor systems. Impairments or an inability to efficiently control and coordinate these motor systems would impact the accuracy of speech production and intelligibility. Speech sound disorders (SSD) are complex behavioural speech disorders in children, referring to deficits in motor speech control of the articulatory mechanisms (i.e., phonetic disorders, childhood apraxia of speech) and/or deficits in the general processing, organization, and cognitive representation of linguistic information. It is likely that children with SSD are a heterogeneous group at many levels, and vary in terms of their severity, error types, causal and maintenance factors. The etiology of most SSD is unknown. Differential diagnosis is often challenging in these children as they may show mixed profiles [1].

Neuroimaging studies have shown that infarcts to the left hemisphere, especially those involving the inferior frontal regions, including Broca's area (e.g., [2] [3]) and the insula (e.g., [4] [5]) have consistently been associated with motor speech planning difficulties in adults. There have been fewer neuroimaging studies examining the neural activities underlie the articulatory control and/or phonological processing in children with SSD. In a group of adolescents with a history of SSD [6], a functional magnetic resonance imaging (fMRI) study examining phonological memory in a non-word repetition task reported a marked right lateralized hypoactivation in inferior frontal and middle temporal gyri for the SSD cohort, suggesting deficits in phonological processing and speech perception. In addition, hyperactivation was observed in bilateral pre-motor and supplementary motor cortices, supramarginal gyrus, inferior parietal lobe, and cerebellum suggesting compensatory increases in cognitive effort and additional recruitment of articulatory rehearsal networks and/or phonologic stores. Similarly, Preston and colleagues [7] studied fMRI responses in children aged 8 to 11 years, whose speech errors had not yet resolved by age 8.5 years, and found that their neural activation patterns to words and non-words differed from that of typically speaking controls. Children with speech errors showed hypoactivation in left middle temporal gyrus and hyperactivation in several cortical (e.g., bilateral parietal regions and left superior temporal gyrus) and subcortical (e.g., globus pallidus and insula) regions relative to controls. More recently, an event-related potentials study involving children aged 9 - 15 years with deficits in motor speech control and planning, compared to control children with typical speech during a picture-naming task. The results showed reduced amplitudes over the right hemisphere prior to the production of multisyllabic relative to monosyllabic words in the children with motor speech control deficits [8]. These studies provided consistent findings indicating different cortical activities related to processing of speech production areas in children with SSD.

Far less investigated are the neurobiological underpinnings of treatment-related 
changes in SSD. Using structural imaging, a study from our group [9] found that young children (aged 3 - 6 years) with idiopathic apraxia (a form of SSD) had thicker left supramarginal gyri than age-matched controls, which subsequent to motor speech intervention, showed thinning to levels comparable to the controls. We suggested that these changes may reflect experience-dependent structural neuroplasticity in areas responsible for sensorimotor integration and phonological processing.

The goal of the current study is to characterize changes in cortical activity associated with participation in a motor-based intervention in children with SSD. We employed Magnetoencephalography (MEG) to investigate the changes in neural dynamics in children with SSD over a course of intensive speech therapy. MEG is a technique that captures task-related neuronal firing with high temporal and spatial resolution [10]. MEG has increasingly been used to investigate the localization [11], lateralization [12] [13], and performance [14] on speech and language tasks in children.

Neuroimaging studies have provided converging evidence that the motor cortex provides a common bilateral structural network for various basic voluntary oromotor tasks [15] [16] suggesting the verbal and non-speech oral movements may share the similar neural pathway during the initial processing stage [17]. Therefore, in this study, we used a non-speech jaw opening task and a simple syllable PA repetition task to observe the cortical activities before and after an eight-week course of motor speech intervention. Along with the acquisition of MEG data, three standardized speech tests were administered to assess motor speech control and articulation as well as a speech acoustic measure for PA productions. We hypothesized that motor speech therapy would result in changes in the functional activity of cortical regions known to support the motor control of speech. Our specific aim was to use MEG to gain insight into the neural mechanisms underlying motor speech control and production.

\section{Methods}

\subsection{Participants}

This study was approved by the Hospital for Sick Children Research Ethics Board. Written informed consent and/or verbal assent was obtained from parents and children, respectively.

In the current study, fourteen children were recruited as participants and completed the intervention. However, two children did not return for post-intervention scanning and three children moved excessively in the MEG for the pre, post or both scans. As a result, nine children with SSD (mean age $=4.2$ years) formed the final analysis cohort (Table 1). Participants were selected from the waiting list for speech therapy at The Speech and Stuttering Institute, Toronto, Canada. The Speech and Stuttering Institute keeps a waitlist for families interested in commencing therapy. For this study, families were contacted over the summer and recruited to this study which began at the same time as the 
Table 1. Participant demographics.

\begin{tabular}{cc}
\hline Mean age & $4.2 \pm 1.13$ years \\
Sex & 7 boys; 2 girls \\
PPVT-3 & $106 \pm 12.38$ \\
EVT-2 & $109 \pm 9.91$ \\
\hline
\end{tabular}

school year. In the absence of known neurological diseases and hearing difficulties, participants selected for the study were confirmed with the diagnosis of moderate to severe oral motor control issues and motor planning deficits (see [18] for a checklist). At the time of recruitment and during the study, none of the children received any additional therapy outside of the study (as reported by caregivers). To confirm that participants' gross language skills were intact and at an age-appropriate level, the Peabody Picture Vocabulary Test, $3^{\text {rd }}$ edition (PPVT-3; [19]) and the Expressive Vocabulary Test, $2^{\text {nd }}$ edition (EVT-2; [20]) were administered prior to the first MEG recording. Participants scored within normal limits across all language measures. For all participants, parents gave written informed consent and children gave verbal assent to participate in the study. Additionally, 15 typically developing control participants (aged $3-4$ years) were recruited from the community, and were negative for a history of developmental speech-language delays, neurological conditions and hearing issues. All completed a first scan and agreed to return 8 - 10 weeks later for a second scan. However, there was a $75 \%$ attrition rate in the control group with only four children returning; there were insufficient data for subsequent analysis.

\subsection{Intervention}

For the children with SSD, a motor speech intervention called PROMPT (Prompts for Restructuring Oral and Muscular Phonetic Targets; [21]) was chosen as it has demonstrated efficacy in treating children with speech sound disorders and speech motor control issues (e.g., [9] [22] [23] [24]). The PROMPT approach is a dynamic tactile and kinesthetic based treatment approach designed to cue or "prompt" correct articulatory positions, movement trajectories (at the phoneme, word, and phrasal level) and movement sequencing to facilitate the production of target sounds in connected speech. In this study, participants received twice-weekly sessions of PROMPT therapy for eight weeks; each session was 45-minute long and parents were assigned ten-minutes of homework to be completed daily with the child. A licensed speech-language pathologist with specialized training in PROMPT (DG) offered all of the treatment sessions. Treatment fidelity was monitored for $20 \%$ of the sessions by using standard procedures that reflect an integration of treatment delivery and clinical skill as a single quantifiable metric (with established inter-rater reliability using Krippendorff's Alpha > 80\%; [25]). 


\subsection{Behavioral Measures of Oral Motor Skills}

As well, three standardized tests were given for all participants before and after the intervention. The first was the Verbal Motor Production Assessment for Children (VMPAC; [26]) which is used to assess the neuromotor integrity of the speech motor system. For the purposes of this study, only the sub-tests most pertinent to volitional oral motor control (that is, the Focal oral motor (VMPAC-FOC) and Sequencing (VMPAC-SEQ) sub-tests) were utilized. The second was the Goldman-Fristoe Test of Articulation, 2nd Edition (GFTA-2; [27]) which is a systematic assessment of a child's articulation of English consonants. The third test was the Hodson Computerized Analysis of Phonological Patterns (HCAPP; [28]) used to measure changes in the child's phonological system by assessing productions for phonological deviations. All assessments were administered within two weeks of the beginning and end of therapy by licensed therapists who were blind to the diagnosis and treatment of each child and conducted at the Speech and Stuttering Institute (Toronto, Canada).

Further, acoustic measurements on voice onset time (VOT) for PA productions before and after speech therapy were analyzed. VOT has been used to reflect subtle motor speech coordination skills where a smaller VOT variability for voiceless stops may reflect a better inter-gestural coordination in children [29] [30].

\subsection{MEG Data Acquisition and Analysis Procedure}

MEG data were acquired with participants lying supine in a CTF 151-channel whole-head MEG system (MISL, Coquitlam BC). MEG signals were acquired continuously, digitized at a sampling rate of $625 \mathrm{~Hz}$ with a $200 \mathrm{~Hz}$ low-pass filter. After completion of the MEG, a co-registered structural MRI was obtained for each subject (T1-weighted sagittal 3D MPRAGE, FOV/Res $=192 \times 240 \times$ $256,1 \mathrm{~mm}$ ISO voxels, TR/TE/TI/FA $=2300 / 2.96 / 900 / 9$, GRAPPA $=2$ ) on a $3 \mathrm{~T}$ scanner with a 12-channel head coil (Siemens Tim Trio, Erlagen, Germany).

Participants visited the MEG Lab twice: once before the intervention, and again, 9 - 10 weeks later, after the intervention. Within the MEG scanner, participants performed an oromotor task and a syllable repetition task. These two tasks were translated directly from our prior investigation of the neural correlates underlying speech and non-speech productions in control adults where we successfully identified involvement of motor-related neural mechanisms for the oromotor task, and language/speech-related neural mechanisms for the syllable repetition task [17]. The oromotor task involved opening and closing their mouth (mouth open-close; MOC) in one complete smooth movement. The syllable PA task involved producing the speech sound /pa/ aloud. Prior to data acquisition, participants practiced each task and were instructed to stay "as still as statues" in the scanner. Movement tolerance during scanning was $5 \mathrm{~mm}$. Participants completed 115 trials of each task in separate conditions. Between trials, subjects fixated on a small white circle on the screen. When the colour of the circle 
changed to green, this was the cue to perform the task. Trials were cued with a variable interstimulus interval of 3.5 - $3.9 \mathrm{sec}$. To help separate intentional movements from spurious movements, all participants wore an ultrasound-emitting device taped to their jaw. This device tracked jaw movement and displacement as an additional analog-to-digital channel on the MEG recording (see [17] for a full description). This recording was visually scanned for unusual deflections, and when seen, that trial was excluded from further processing.

Continuous MEG data were epoched into trials $(-1.0 \mathrm{sec}$ to $+1.0 \mathrm{sec})$ time-locked to the presentation of the visual cue. Epoched data were analysed using a free, downloadable source reconstruction packaged called Brainwave (http://cheynelab.utoronto.ca/brainwave). Data were band pass filtered from 1 to $40 \mathrm{~Hz}$ and a non-overlapping, sliding window approach was taken with nine 50 ms windows from 100 - $550 \mathrm{~ms}$ (i.e., 100 - $150 \mathrm{~ms}, 150$ - 200, 200 - 250, 250 300, 300 - 350, 350 - 400, 400 - 450, 450 - 500 and 500 - $550 \mathrm{~ms}$ ) and submitted to event-related beamforming [31] over integrated time windows to identify neural sources active in each of these windows.

Pre- and post-intervention clinical test scores and acoustic analysis measures were statistically compared with paired-samples t-tests. Brain magnitude and latency data were obtained from reconstructed virtual waveforms and submitted to permutation testing using a single-threshold maximal statistic, which accounts for multiple comparisons.

\section{Results}

All participants exhibited moderate to severe oral motor control issues on the VMPAC with primary difficulties involving jaw and orofacial control, including decreased jaw stability, limited control of the degree of jaw range of motion (overshoot/overextension), decreased lip retraction and overly retracted lips. Thus the goals were increased jaw control, decreased overall excursion, improved midline control, and better facilitation of jaw grading for speech production, such as for bilabial speech sounds. Results from the standardized testing are summarized in Table 2 which showed gains on all measures as tested with paired samples t-tests: VMPAC-FOC ( $t=5.94, p<0.001$; Cohen's $d=1.0)$; VMPAC-SEQ ( $\mathrm{t}=4.82, p<0.001$; Cohen's $d=0.9)$; GFTA-2 $(\mathrm{t}=2.38, p<0.05$; Cohen's $d=0.7)$ and HCAPP $(t=-5.84, p<0.001$; Cohen's $d=0.9)$. These indicate a positive effect of the intervention on motor speech skills and articulation.

Acoustic analyses demonstrated an increase in the mean VOT from pre-intervention (mean \pm SD: $63.3 \mathrm{~ms} \pm 39.0)$ to post-intervention $(79.5 \pm 33.7)$. Coefficient of variance (CoV) decreased from pre (61.6\%) to post (42.4\%).

The MEG analysis program provides the timing, Talairach coordinates, anatomical and Brodmann area labels for all brain areas that were significantly different before and after the intervention $(p<0.025)$ for both MOC and PA tasks. These are summarized in Table 3 and the spatiotemporal progression of these significant activations are visualized in Figure 1. Figure 2 contains the 3-dimensional 
Table 2. Standardized scores for VMPAC-FOC and VMPAC-SEQ subtest, GFTA-2 and HCAPP at PRE-intervention (PRE) and POST-intervention (POST) testing for each participant.

\begin{tabular}{ccccccccc}
\hline \multirow{2}{*}{ Participant } & \multicolumn{2}{c}{ VMPAC-FOC } & \multicolumn{2}{c}{ VMPAC-SEQ } & \multicolumn{2}{c}{ GFTA-2 } & \multicolumn{2}{c}{ HCAPP } \\
\cline { 2 - 8 } & PRE & POST & PRE & POST & PRE & POST & PRE & POST \\
\hline P1 & 69 & 83 & 63 & 83 & 66 & 66 & 214 & 163 \\
P2 & 67 & 78 & 81 & 87 & 40 & 54 & 104 & 36 \\
P3 & 50 & 61 & 30 & 48 & 65 & 90 & 130 & 64 \\
P4 & 55 & 69 & 49 & 70 & 58 & 70 & 55 & 32 \\
P5 & 63 & 71 & 54 & 61 & 69 & 70 & 106 & 82 \\
P6 & 69 & 83 & 63 & 83 & 82 & 79 & 84 & 61 \\
P7 & 77 & 81 & 71 & 80 & 72 & 87 & 102 & 78 \\
P8 & 76 & 78 & 73 & 80 & 73 & 69 & 112 & 92 \\
P9 & 82 & 87 & 73 & 74 & 64 & 84 & 117 & 75 \\
\hline
\end{tabular}

Table 3. Timing, coordinates and anatomical labels for regions showing significant pre-post differences in the two task conditions.

\begin{tabular}{|c|c|c|c|c|c|c|}
\hline & Time & Value & $\mathrm{X}$ & $\mathrm{Y}$ & $\mathrm{Z}$ & Talairach Location (Brodmann Area) \\
\hline \multirow{12}{*}{$\begin{array}{c}\text { Mouth } \\
\text { Open-Close }\end{array}$} & $100-150$ & 2.22 & -32 & 9 & 33 & L precentral gyrus (BA9) \\
\hline & $150-200$ & 1.91 & -44 & 27 & -5 & L inferior frontal gyrus (BA47) \\
\hline & & 1.87 & 51 & -42 & 6 & $\mathrm{R}$ middle temporal gyrus (BA21) \\
\hline & & 1.84 & -44 & 24 & 21 & L pre-central gyrus (BA6) \\
\hline & $200-250$ & 2.24 & 40 & 12 & 10 & $\mathrm{R}$ insula (BA13) \\
\hline & $250-300$ & 2.09 & 48 & -41 & 32 & $\mathrm{R}$ supramarginal gyrus (BA40) \\
\hline & $300-350$ & 2.04 & -51 & -31 & -5 & $\mathrm{~L}$ middle temporal gyrus (BA21) \\
\hline & $350-400$ & 2.06 & -32 & 28 & 17 & L insula (BA13) \\
\hline & & 2.02 & -55 & -19 & 8 & L superior temporal gyrus (BA41) \\
\hline & $400-450$ & 2.08 & -59 & -8 & 4 & L superior temporal gyrus (BA22) \\
\hline & $450-500$ & 2.32 & 55 & -26 & 23 & $\mathrm{R}$ inferior parietal gyrus (BA40) \\
\hline & $500-550$ & 2.12 & -51 & 24 & 6 & L inferior frontal gyrus (BA45) \\
\hline \multirow[t]{9}{*}{$\mathrm{PA}$} & $100-150$ & & & & & \\
\hline & $150-200$ & 0.86 & -48 & -42 & 13 & $\mathrm{~L}$ middle temporal gyrus (BA21) \\
\hline & $200-250$ & 0.93 & -42 & 17 & 17 & R superior temporal gyrus/insula (BA13) \\
\hline & $250-300$ & & & & & \\
\hline & $300-350$ & & & & & \\
\hline & $350-400$ & 1.18 & 40 & 23 & 3 & $\mathrm{R}$ inferior frontal gyrus (BA45) \\
\hline & $400-450$ & & & & & \\
\hline & $450-500$ & & & & & \\
\hline & $500-550$ & & & & & \\
\hline
\end{tabular}




\begin{tabular}{|c|c|c|c|c|c|c|c|c|c|c|}
\hline \multicolumn{2}{|c|}{ mouth open-close } & $100-150$ & $150-200$ & $200-250$ & $250-300$ & $300-350$ & $350-400$ & $400-450$ & $450-500$ & $500-550$ \\
\hline \multirow{4}{*}{ LEFT } & frontal & $\rightarrow$ & $\uparrow \mathrm{BA} 47^{*}$ & $\rightarrow$ & $\rightarrow$ & $\rightarrow$ & $\rightarrow$ & $\rightarrow$ & $\rightarrow$ & BA45 \\
\hline & motor & $\uparrow \downarrow \mathrm{BA9}$ & $\uparrow \downarrow B A 6 *$ & ..... & ..... & $\ldots .$. & ..... & $\ldots .$. & ..... & ...... \\
\hline & temporal & $\rightarrow$ & $\rightarrow$ & $\rightarrow$ & $\rightarrow$ & $\downarrow \mathrm{BA} 21$ & $\downarrow$ BA41 * & $* \quad \downarrow B A 22$ & ..... & ..... \\
\hline & insula & $\rightarrow$ & $\rightarrow$ & $\rightarrow$ & $\rightarrow$ & $\rightarrow$ & $\uparrow \mathrm{BA} 13 *$ & * $\quad \ldots .$. & $\ldots .$. & ..... \\
\hline \multirow{4}{*}{ RIGHT } & frontal & $\ldots .$. & $\ldots .$. & $\ldots .$. & $\ldots .$. & $\ldots .$. & $\ldots .$. & $\ldots .$. & $\ldots .$. & $\ldots .$. \\
\hline & motor & ..... & $\ldots .$. & ..... & $\ldots .$. & $\ldots .$. & ..... & ..... & ..... & ..... \\
\hline & temporal & $\rightarrow$ & $\uparrow \downarrow \mathrm{BA} 21$ & $\rightarrow$ & $\uparrow \downarrow \mathrm{BA} 40$ & $\rightarrow$ & $\rightarrow$ & $\rightarrow$ & $\uparrow \downarrow \mathrm{BA} 40$ & ..... \\
\hline & insula & $\rightarrow$ & $\rightarrow$ & $\approx B A 13$ & $\ldots .$. & $\ldots .$. & $\ldots .$. & $\ldots .$. & $\ldots .$. & $\ldots .$. \\
\hline \multicolumn{11}{|l|}{ PA } \\
\hline \multirow{4}{*}{ LEFT } & frontal & ...... & $\ldots .$. & $\ldots .$. & ...... & ...... & $\ldots \ldots$ & $\ldots .$. & ...... & ...... \\
\hline & motor & $\ldots .$. & $\ldots .$. & $\ldots .$. & $\ldots .$. & $\ldots .$. & $\ldots .$. & $\ldots .$. & $\ldots .$. & $\ldots .$. \\
\hline & temporal & $\rightarrow$ & $\uparrow \mathrm{BA} 21$ * & $\ldots .$. & $\ldots .$. & $\ldots .$. & ..... & ..... & ..... & ..... \\
\hline & insula & $\ldots .$. & $\ldots .$. & $\ldots .$. & $\ldots .$. & $\ldots$. & $\ldots .$. & $\ldots .$. & $\ldots .$. & $\ldots .$. \\
\hline \multirow{4}{*}{ RIGHT } & frontal & $\rightarrow$ & $\rightarrow$ & $\rightarrow$ & $\rightarrow$ & $\rightarrow$ & $\downarrow$ BA45 * & $\ldots .$. & $\ldots .$. & $\ldots .$. \\
\hline & motor & ..... & ..... & $\ldots .$. & $\ldots .$. & ..... & ..... & ..... & ..... & ..... \\
\hline & temporal & ..... & ..... & $\ldots$. & $\ldots .$. & $\ldots .$. & ..... & ..... & $\ldots .$. & ..... \\
\hline & insula & $\rightarrow$ & $\rightarrow$ & $\approx \mathrm{BA} 13 *$ & $\ldots .$. & $\ldots .$. & $\ldots .$. & $\ldots .$. & $\ldots .$. & $\ldots .$. \\
\hline
\end{tabular}

Figure 1. Brodmann Area labels for areas showing significant changes by task and time-window. An "up" arrow ( $\uparrow$ ) indicates an increase in activation in that area. A "down" arrow $(\downarrow)$ indicates a decrease in activation in that area. A pair of arrows ( $\downarrow$ ) indicates an initial increase followed by a subsequent decrease. The reverse situation of an initial decrease followed by an increase was never seen. The equivocal symbol $(\approx)$ indicates that while the statistical comparison was significant, investigation of the virtual sensor did not clearly indicate a direction. An asterisk $\left(^{*}\right)$ indicates that the virtual sensor from this region is included in Figure 3 .

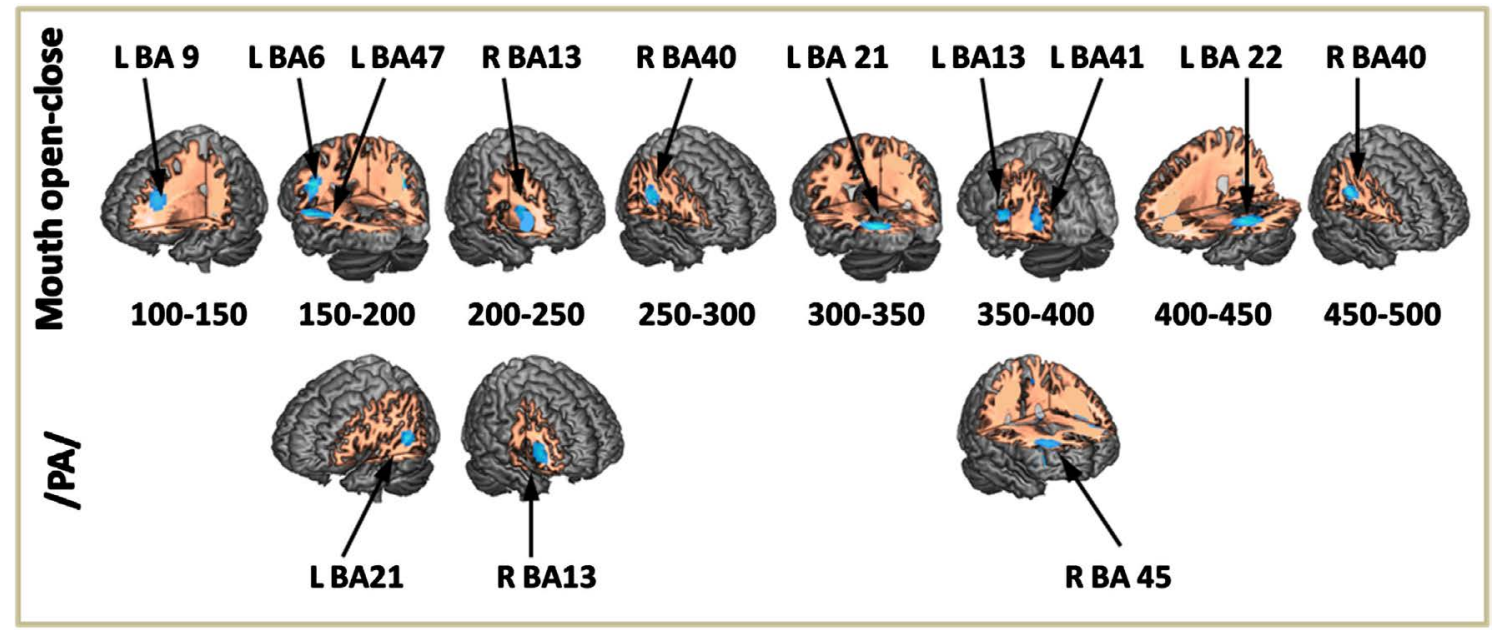

Figure 2. Three-dimensional brain images showing the areas that were significantly different between the pre- and post-intervention conditions for both the MOC task and the /pa/ production task.

brain images for areas that were significantly different between pre- and post-intervention indicated.

To further explore the timing of differences in brain areas as a function of speech intervention, we reconstructed virtual sensors which demonstrate the time course of activations, pre and post intervention, for each condition. These are shown in Figure 3. In this figure, the blue line indicates the magnitude of 


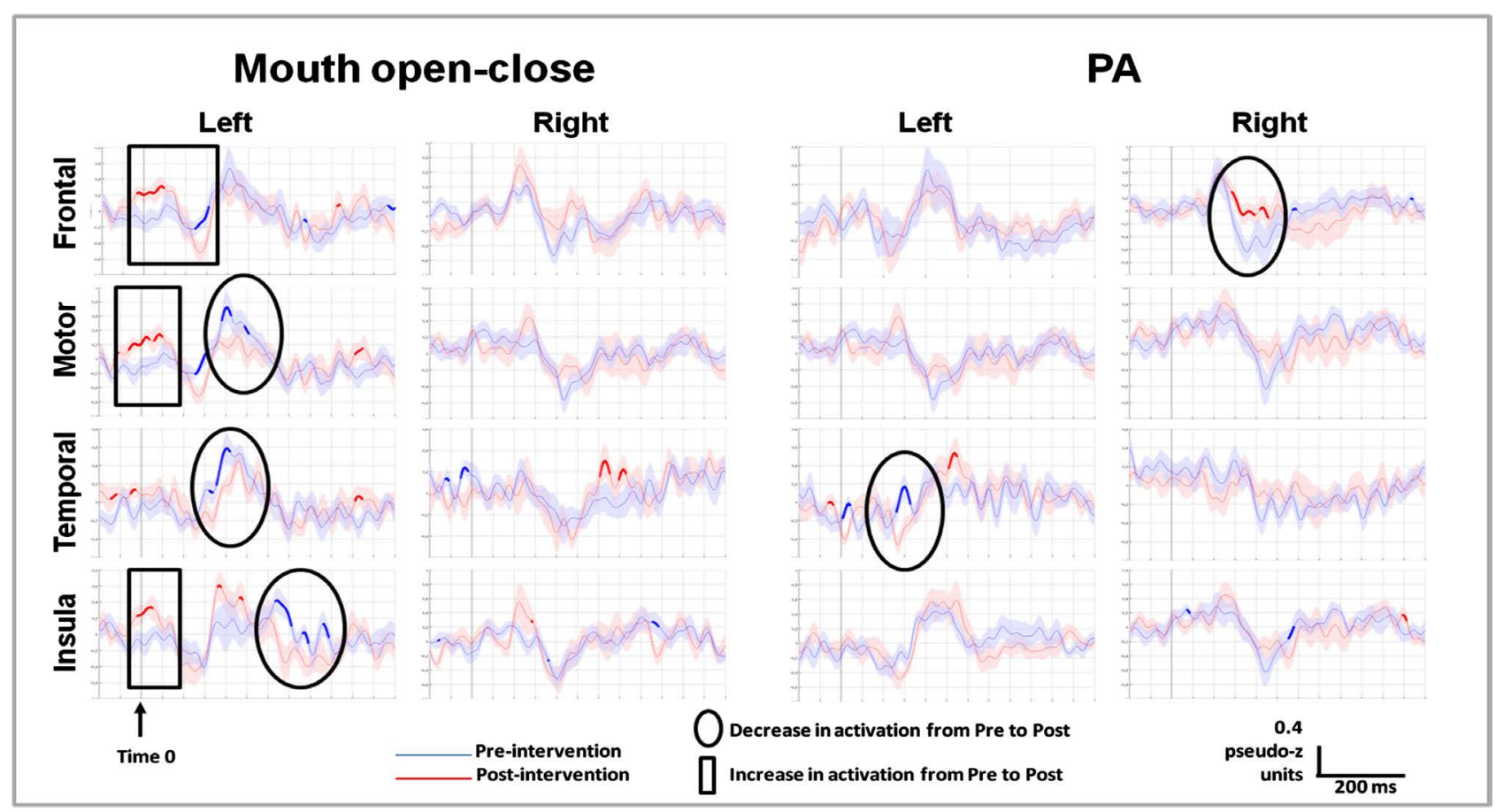

Figure 3. Virtual sensors for each task.

brain activations in the PRE condition and the red line shows the magnitude of activations in the POST condition. Sections where the lines are thicker indicate time points when the pre and post conditions are significantly different. Significant differences are always marked on the upper line; thus Table 3 also indicates the direction of change in each brain region. Only changes reaching statistical significance, and sustained for $>50 \mathrm{~ms}$ (the duration of our beamforming window) are interpreted and these are identified by a box for an increase in activation and a circle when there is a decrease in activation. The most striking result is the reproducibility of the tracings despite being recorded 9 to 10 weeks apart. This attests to the technical quality of the MEG recording [32].

In the oromotor (Mouth open-close) task (Figure 3, left panel), the contrasts indicate that all sustained, significant changes occurred in the left hemisphere. The POST condition had significantly higher activations, early in the response, in the frontal cortex (inferior frontal gyrus, BA 44/45), motor cortex (precentral gyrus, BA 6) and insula (BA 13); whereas, later in the response, there was a decrease in activation in the motor cortex, temporal cortex (superior temporal gyrus, BA 41/22) and insula. In the speech (PA) task (Figure 3, right panel), surprisingly, there are few differences, and the contrasts indicate reduced activations in the right frontal cortex (inferior frontal gyrus, BA 45) and the left temporal cortex which included the supramarginal gyrus (BA 40) and middle temporal gyrus (BA 21).

\section{Discussion}

This is the first study to use MEG to investigate neural changes in children with 
SSD who underwent a brief intensive motor speech intervention. Using two simple but different motor speech tasks (i.e., MOC and PA production) which we have tested in control adults, we were able to identify changes in specific responses in brain areas that may be associated with motor speech control. In conjunction with the MEG analysis, clinical assessments of motor speech skills were acquired which indicated a positive effect on motor speech control and thus provide preliminary support for the possible benefits of PROMPT intervention in children with SSD. Over the course of eight-weeks of intensive therapy, our MEG study showed changes in the functional activation of cortical regions related to motor speech control and speech production that are in line with the behavioral data from the motor and speech assessments.

With regards to the neural correlates of non-speech oromotor control, we were able to use virtual sensor analyses to contrast differences in the timing and magnitude of activations generated by mouth open-close movements before and after PROMPT intervention (Figure 2, left panel). Our data showed marked differences in brain activation in several regions associated with oromotor control. The most striking observation is that the majority of changes are in the left hemisphere, where the impact of therapy is characterized by an early increase followed by a later decrease in activation.

While each individual virtual sensor could be interpreted separately, it is the overall pattern of findings that tells the story. There are two time windows where changes occur in concert across sensors. The first is around time zero, and the second is around $200 \mathrm{~ms}$. At time zero, across the frontal, motor and insular sensors, there is greater activation post-intervention. This time zero activation is surprising as it occurs prior to the presentation of the visual stimulus, and suggests that participants may be anticipating the task and possibly preparing earlier; thus, resulting in the production of more consistent responses. The later decrease in activation suggests they rely less on these areas and neural and response preparation is subsumed earlier. An alternative explanation is that these differences are attributable to the participants' greater familiarity with the task during the second MEG session; however, the participants also showed significantly improved scores on standardized testing and significant changes on acoustic analysis of voice-onset time. Taken together, there is strong suggestion that there is a relation between the clinical and acoustic improvements and the observed brain changes.

An interesting post-intervention change of note is in the left insula in the 200 $300 \mathrm{~ms}$ time window. Here, visual inspection shows that this region has much higher activation post-therapy; while the peaks reach statistical significance, our stringent statistical criteria do not show a sustained significant change. However, there is evidence that one important role of the insula is in motor control and articulatory planning (e.g., [4] [5] [17] [33] [34]). Our finding of greater activation in the left insula along with earlier activations in frontal and motor areas following intervention may be a reflection of better oromotor coordination and 
control.

For the MOC condition, we also saw a sustained decrease of activation in primary auditory (BA 21) and association areas (BA 41, BA 22) in the $300-450$ time range. These areas typically play a role in language comprehension and thus we did not expect to see their involvement in this task. We speculate that the child may be verbally "coaching" him/herself through the task in the "pre" condition, and with increased familiarity with the task or perhaps with better motor control, this "coaching" is not as essential in the "post" condition. This hypothesis requires further testing.

For the PA production task, in the left hemisphere, the activations are well-formed, clear and reproducible in the frontal, motor and insular regions. The other significant change is in the left temporal region and shows a decrease in activation post-intervention. However, when looking at the entire waveform, it is clear that while the decrease is sustained and reaches statistical significance, the overall pattern is one where the waveform shows a clearer set of peaks post-intervention. In the neurophysiology literature, this is interpreted as improved synchronization of the neurons activated in this region. This is of interest as studies show the left middle temporal gyrus to play an important role in speech comprehension and production [35], with a recent meta-analysis confirming its role in both functions [36]. Thus, this finding of more synchronized activity in the temporal regions post-intervention may provide additional reinforcement of improved perception of speech-related functions which is key to successful speech production.

With regards to the right hemisphere, the frontal region showed significantly less activation post-intervention. The homologous language region in the right hemisphere has been found to activate in situations where additional support is required to process speech and language, for example in young children, or in some clinical conditions. The finding here of decreased activation post-intervention suggests less reliance on this region, and possibly a more efficient, mature response overall.

One striking feature from the PA recordings is the similarity and reproducibility of the waveforms before and after intervention which is more than nine weeks apart. This overall finding of few differences pre- and post-intervention on the PA task may be due to the fact that the /pa/ phoneme is already well-learned in our group. Reviewing the treatment goals for each participant, only two had treatment goals that included increased labio-facial control for lip movements involving /p/. It may be that MEG, with its high spatial temporal resolution, is able to capture the brain changes associated with very subtle improvements, and MEG resolution is high enough to be specific to the task applied. This would suggest that future studies need to adjust the stimulation parameters to fit the particular difficulties experienced by each child.

Finally, our MEG results are supported by our acoustic findings. Measuring VOT has been used in developmental studies to indicate the mastery of speech 
gestural coordination. During typical motor speech development, children exhibit a shorter and more variable VOT relative to adults, and with development, the VOT increases and becomes less variable (e.g., [29] [30] [37] [38]). This is the pattern seen in the current study. After PROMPT therapy, the mean VOT is increased and $\mathrm{CoV}$ is decreased.

\section{Conclusions}

In summary, we found a positive outcome effect of PROMPT on motor speech control and articulation for children with SSD, and we observed dynamic changes in associated brain regions after an eight-week course of intensive motor speech therapy. Using a pre-post design with a brief, intense intervention, there is good evidence that participation in the therapy program drove the observed functional changes in the regions associated with oromotor control and speech production. This is an important area of study as it suggests a mechanism of therapeutic action that underlies behavioral change. Understanding mechanisms of action has immediate ramifications for improving service delivery (treatment dosage, intensity \& delivery method) but also demonstrates treatment efficacy which facilitates accountability with stakeholders and improves "buy-in" from parents.

For future research, children with SSD who do not undergo treatment (waitlist group) or an age-matched control group with no speech and language issues should be recruited to act as a control group. This will allow us to tease apart treatment versus maturation changes in neurophysiological processes. Another possible design would be to incorporate three time points, so as to ascertain whether treatment gains are sustained and retained. Future research would also benefit from the inclusion of different levels of stimulus complexity, as well as specific tasks targeted for each child's therapy goals. The former suggestion would provide further insight into the theoretical aspects of phonological processing, motor planning and articulatory control during speech preparation in SSD, possibly leading to the identification of neurophysiology markers of subtypes of speech impairment during speech production. The latter would allow the development of individualized treatment plans that can be tracked or monitored for efficacy. While there are many opportunities for future research, this first study using MEG demonstrates the value of this neuroimaging modality to capture subtle brain functional changes, and its feasibility for testing young children with clinical conditions, including SSD.

\section{Acknowledgements}

This work was funded by a Canadian Institutes of Health Research operating grant (CIHR MOP-89961) to the last two authors, LDN and EWP. The authors would like to acknowledge Matt J. MacDonald, Anna Oh, Sarah Vinette, Gordon Hua and Marc Lalancette for participation in data acquisition and data analysis. 


\section{References}

[1] Strand, E.A. and McCauley, R.J. (2008) Differential Diagnosis of Severe Speech Impairment in Young Children. The ASHA Leader, 13, 10-13. https://doi.org/10.1044/leader.FTR1.13102008.10

[2] Hillis, A.E., Work, M., Barker, P.B., Jacobs, M.A., Breese, E.L. and Maurer, K. (2004) Reexamining the Brain Regions Crucial for Orchestrating Speech Articulation. Brain, 127, 1479-1487. https://doi.org/10.1093/brain/awh172

[3] Jordan, L.C. and Hillis, A.E. (2006) Disorders of Speech and Language: Aphasia, Apraxia and Dysarthria. Current Opinion in Neurology, 19, 580-585. https://doi.org/10.1097/WCO.0b013e3280109260

[4] Dronkers, N.F. (1996) A New Brain Region for Coordinating Speech Articulation. Nature, 384, 159-161. https://doi.org/10.1038/384159a0

[5] Nagao, M., Takeda, K., Komori, T., Isozaki, E. and Hirai, S. (1999) Apraxia of Speech Associated with an Infarct in the Precentral Gyrus of the Insula. Neuroradiology, 41, 356-357. https://doi.org/10.1007/s002340050764

[6] Tkach, J.A., Chen, X., Freebairn, L.A., Schmithorst, V.J., Holland, S.K. and Lewis, B.A. (2011) Neural Correlates of Phonological Processing in Speech Sound Disorders: A Functional Magnetic Resonance Imaging Study. Brain and Language, 119, 42-49. https://doi.org/10.1016/j.bandl.2011.02.002

[7] Preston, J.L., Felsenfeld, S., Frost, S.J., Mencl, W.E., Fulbright, R.K., Grigorenko, E.L., Lan-di, N., Seki, A. and Pugh, K.R. (2012) Functional Brain Activation Differences in School-Age Children with Speech Sound Errors: Speech and Print Processing. Journal of Speech, Language and Hearing Research, 55, 1068-1082. https://doi.org/10.1044/1092-4388(2011/11-0056)

[8] Preston, J.L., Molfese, P.J., Gumkowski, N., Sorcinelli, A., Harwood, V., Irwin, J.R. and Landi, N. (2014) Neurophysiology of Speech Differences in Childhood Apraxia of Speech. Developmental Neuropsychology, 39, 385-403. https://doi.org/10.1080/87565641.2014.939181

[9] Kadis, D.S., Goshulak, D., Namasivayam, A., Pukonen, M., Kroll, R., De Nil, L.F., Pang, E.W. and Lerch, J.P. (2014) Cortical Thickness in Children Receiving Intensive Therapy for Idopathic Apraxia of Speech. Brain Topography, 27, 240-247. https://doi.org/10.1007/s10548-013-0308-8

[10] Hari, R. and Salmelin, R. (2012) Magnetoencephalography: From SQUIDS to Neuroscience. Neuroimage 20th Anniversary Special Edition. Neuroimage, 6, 386-396. https://doi.org/10.1016/j.neuroimage.2011.11.074

[11] Kadis, D.S., Smith, M.L., Mills, T. and Pang, E.W. (2008) Expressive Language Mapping in Children Using MEG. Down Syndrome Quarterly, 10, 5-12.

[12] Kadis, D.S., Pang, E.W., Mills, T., Taylor, M.J., McAndrews, M.P. and Smith, M.L. (2011) Characterizing the Normal Developmental Trajectory of Expressive Language Lateralization Using MEG. Journal of the International Neuropsychological Society, 17, 896-904. https://doi.org/10.1017/S1355617711000932

[13] Ressel, V., Wilke, M., Lidzba, K., Lutzenberger, W. and Krageloh-Mann, I. (2008) Increases in Language Lateralization in Normal Children as Observed Using Magnetoencephalography. Brain and Language, 106, 167-176.

https://doi.org/10.1016/j.bandl.2008.01.004

[14] Kikuchi, M., Shitamichi, K., Yoshimura, Y., Ueno, S., Remijn, G.B., Hirosawa, T., Munesue, T., Tsubokawa, T., Haruta, Y., Oi, M., Higashida, H. and Minabe, Y. (2011) Lateralized Theta Wave Connectivity and Language Performance in 2- to 
5-Year-Old Children. The Journal of Neuroscience, 31, 14984-14988. https://doi.org/10.1523/JNEUROSCI.2785-11.2011

[15] Salmelin, R. and Sams, M. (2002) Motor Cortex Involvement during Verbal versus Nonverbal Lip and Tongue Movements. Human Brain Mapping, 16, 81-91. https://doi.org/10.1002/hbm.10031

[16] Simonyan, K., Ostuni, J., Ludlow, C.L. and Horwitz, B. (2009) Functional but Not Structural Networks of the Human Laryngeal Motor Cortex Show Left Hemispheric Lateralization during Syllable but Not Breathing Production. The Journal of Neuroscience, 29, 14912-14923. https://doi.org/10.1523/JNEUROSCI.4897-09.2009

[17] Memarian, N., Ferrari, P., Macdonald, M.J., Cheyne, D., De Nil, L.F. and Pang, E.W. (2012) Cortical Activity during Speech and Non-Speech Oromotor Tasks: A Magnetoencephalography (MEG) Study. Neuroscience Letters, 527, 34-39.

https://doi.org/10.1016/j.neulet.2012.08.030

[18] Namasivayam, A.K., Pukonen, M., Goshulak, D., Hard, J., Rudzicz, F., Rietveld, T., Maassen, B., Kroll, R. and Van Lieshout, P. (2015) Treatment Intensity and Childhood Apraxia of Speech. International Journal of Language \& Communication Disorders, 50, 529-546. https://doi.org/10.1111/1460-6984.12154

[19] Dunn, M. and Dunn, L.M. (2007) Peabody Picture Vocabulary Test-3. American Guidance Service, Circle Pines, MN.

[20] Williams, K.T. (1997) Expressive Vocabulary Test. American Guidance Services, Circle Pines, $\mathrm{MN}$.

[21] Chumpelik, D.A. (1984) The PROMPT System of Therapy: Theoretical Framework and Applications for Developmental Apraxia of Speech. Seminars in Speech and Language, 5, 139-156. https://doi.org/10.1055/s-0028-1085172

[22] Dale, P. and Hayden, D. (2013) Treating Speech Subsystems in CAS with Tactual Input: The PROMPT Approach. American Journal of Speech-Language Pathology, 22, 644-661. https://doi.org/10.1044/1058-0360(2013/12-0055)

[23] Grigos, M.I., Hayden, D. and Eigen, J. (2010) Perceptual and Articulatory Changes in Speech Production Following PROMPT Treatment. Journal of Medical Speech-Language Pathology, 18, 46-53.

[24] Square, P.A., Namasivayam, A.K., Bose, A., Goshulak, D. and Hayden, D. (2014) Multi-Sensory Treatment for Children with Developmental Motor Speech Disorders. International Journal of Language \& Communication Disorders, 49, 527-542. https://doi.org/10.1111/1460-6984.12083

[25] Hayden, D., Namasivayam, A.K. and Ward, R. (2015) The Assessment of Fidelity in a Motor Speech Treatment Approach. Speech, Language and Hearing, 18, 30-38. https://doi.org/10.1179/2050572814Y.0000000046

[26] Hayden, D. and Square, P. (1999) Verbal Motor Production Assessment for Children. The Psychological Corporation, San Antonio, TX.

[27] Goldman, R. and Fristoe, M. (2000) Goldman-Fristoe Test of Articulation-2. American Guidance Service, Circle Pines, MN.

[28] Hodson, B.W. (2003) Hodson Computerized Analysis of Phonological Patterns. PhonoComp Software (Computer Software), Wichita, KS.

[29] Whiteside, S.P., Dobbin, R. and Henry, L. (2003) Patterns of Variability in Voice Onset Time: A Developmental Study of Motor Speech Skills in Humans. Neuroscience Letters, 347, 29-32. https://doi.org/10.1016/S0304-3940(03)00598-6

[30] Yu, V., De Nil, L.F. and Pang, E.W. (2015) Effects of Age, Sex and Syllable Structure on Voice Onset Time: Evidence from Children's Voiceless Aspirated Stops. Lan- 
guage and Speech, 58, 152-167. https://doi.org/10.1177/0023830914522994

[31] Quraan, M.A. and Cheyne, D. (2010) Reconstruction of Correlated Brain Activity with Adaptive Spatial Filters in MEG. Neurolmage, 49, 2387-2400.

https://doi.org/10.1016/j.neuroimage.2009.10.012

[32] American Clinical Neurophysiology Society (2006) Guideline 9A: Guidelines on Evoked Potentials. Journal of Clinical Neurophysiology, 23, 125-137. https://doi.org/10.1097/00004691-200604000-00010

[33] Cereda, C., Ghika, J., Maeker, P. and Bogousslavsky, J. (2002) Strokes Restricted to the Insular Cortex. Neurology, 59, 1950-1955. https://doi.org/10.1212/01.WNL.0000038905.75660.BD

[34] Murphy, K., Corfield, D.R., Guz, A., Fink, G.R., Wise, R.J.S., Harrison, J. and Adams, L. (1997) Cerebral Areas Associated with Motor Control of Speech in $\mathrm{Hu}-$ man. Journal of Applied Physiology, 83, 1438-1447. https://doi.org/10.1152/jappl.1997.83.5.1438

[35] Hickok, G. and Poeppel, D. (2015) Neural Basis of Speech Perception. Handbook of Clinical Neurology, 129, 149-160. https://doi.org/10.1016/B978-0-444-62630-1.00008-1

[36] Silbert, L.J., Honey, C.J., Simony, E., Poeppel, D. and Hasson, U. (2014) Couple Neural Systems Underlie the Production and Comprehension of Naturalistic Narrative Speech. Proceedings of the National Academy of Sciences of the United States of America, 111, E4687-E4696. https://doi.org/10.1073/pnas.1323812111

[37] Barton, D. and Macken, M.A. (1980) An Instrumental Analysis of the Voicing Contrast in Word-Initial Stops in the Speech of Four-Year-Old English-Speaking Children. Language and Speech, 23, 159-169. https://doi.org/10.1177/002383098002300203

[38] Macken, M.A. and Barton, D. (1980) The Acquisition of the Voicing Contrast in English: A Study of Voice Onset Time in Word-Initial Stop Consonants. Journal of Child Language, 7, 41-74. 